\title{
FORMATION OF MnBi BY MECHANICAL ALLOYING
}

\author{
A. Szlafierek and A. Wrzeciono \\ Institute of Molecular Physics, Polish Academy of Sciences \\ Smoluchowskiego 17, 60-179 Poznań, Poland
}

\begin{abstract}
In this report we demonstrate that mechanical alloying is an alternative process to produce the intermetallic compound $\mathrm{MnBi}$. Magnetically $\mathrm{MnBi}$ powder is prepared from the elemental powders by mechanical alloying in a planetary ball mill and then solid-state reaction at a relatively low temperature. The $\mathrm{MnBi}$ powder was aligned in a magnetic field and isostatic pressed. The X-ray pattern of powder reacted clcarly shows the intensity peaks of the $\mathrm{MnBi}$ phase. After annealing the magnetization was about $1.0 \times 10^{-4} \mathrm{~T} \mathrm{~m}^{3} / \mathrm{kg}$.
\end{abstract}

PACS numbers: 75.50.Kj, 75.50.Tt, 81.20.Ev

\section{Introduction}

Many authors have investigated the magnetic properties of the phase in the Mn-Bi systems. As can be seen from the pliase diagram [1] the phase cannot be prepared directly from a melt of the desired composition. Instead, they are prepared by low-temperature diffusion of elemental powders, or by vapor deposition techniques or by controlled growth of a solid from a Bi rich melt.

After Notis et al. [2] an alloy containing about 2 at\% $\mathrm{Mn}$ and 98 at\% $\mathrm{Bi}$ should solidify at $503 \mathrm{~K}$ to give an eutectic constant of about 3 vol\% ferromagnetic $\mathrm{MnBi}$ in a matrix of diamagnetic $\mathrm{Bi}$.

Whereas in conventional alloying the mixing of the elements takes place in the melt and the solid alloy is formed by solidification, mechanical alloying uses an interdiftusional reaction [3], which is possible due to the formation of ultrafine layered composite particles during the milling process in a ball mill.

Several authors have reported amorphization during ball-milling of powder mixtures of immiscible elements. Amorphization by ball-milling, of elemental mixtures with positive heat of mixing $\left(\Delta H_{\text {mix }}>0\right)$ has been attributed to the increase in the free energy of the elemental powders due to a very high defect density generated by repeated low-temperature deformation. It has been suggested that this energy is mainly stored in a large grain boundary density (as a nanocrystalline structure emerges from the dislocation cell structures of heavily sheared zones). 
The metal powder particles are trapped by the colliding balls, heavily deformed and cold welded leading to characteristically layered particles. Depending on the thermodynamics of the alloy system, on the mechanical workability of the starting powders and on the milling conditions, the interdiflusional reaction to form the alloy can either take place during the milling or during a following heat treatment.

\section{Experimental}

The starting materials for this study were $99.9 \%$ pure $\mathrm{Mn}$ and $\mathrm{Bi}$ powders of particle size $45 \mu \mathrm{m}$. The powders were mechanically alloyed in a high energy mill (Fritsch "Pulverisette 5") under an argon atmosphere for $120 \mathrm{~h}$. The powders were alignment in a magnetic field of $1.6 \mathrm{~T}$ parallel to the cylindrical axis and isostatic pressed under a pressure of $500 \mathrm{MPa}$. A pressed sample was heated in atmosphere of argon at $533 \mathrm{~K}$ for 20 minutes. The samples were cylindrical $3.2 \mathrm{~mm}$ in diameter and $6.3 \mathrm{~mm}$ in length. The density of specimens varied from 6.6 to $6.7 \mathrm{~g} / \mathrm{cm}^{3}$. The crystal structures of the samples were determined by X-ray diffraction with $\mathrm{Cr} K_{\alpha}$ radiation. Magnetization measurements were carried out in the temperature range from $300 \mathrm{~K}$ to $700 \mathrm{~K}$ and in an applied magnetic field up to $9.5 \times 10^{5} \mathrm{~A} / \mathrm{m}$ using a Faraday balance. The virtual Curie point, was obtained by extrapolating the $\sigma$ vs. $T$. The initial susceptibility was measured in an alternating field of $40 \mathrm{~A} / \mathrm{m}$ at $100 \mathrm{IIz}$ by means of a bridge of mutual inductance of the IIartshorn type. A main grain size was estimated from the line broadening of the peak by the Sherrers method. The grain size was estimated to be approximately $80 \mathrm{~nm}$.

\section{Results and discussion}

Figure 1 shows the $\mathrm{X}$-ray powder spectra for alignment and pressed sample. The curves $c$ and $d$ in Fig. 1 show the effect of heat treatment on the diffraction patterns of materials milled for the time $120 \mathrm{~h}$. The patterns before milling (curves

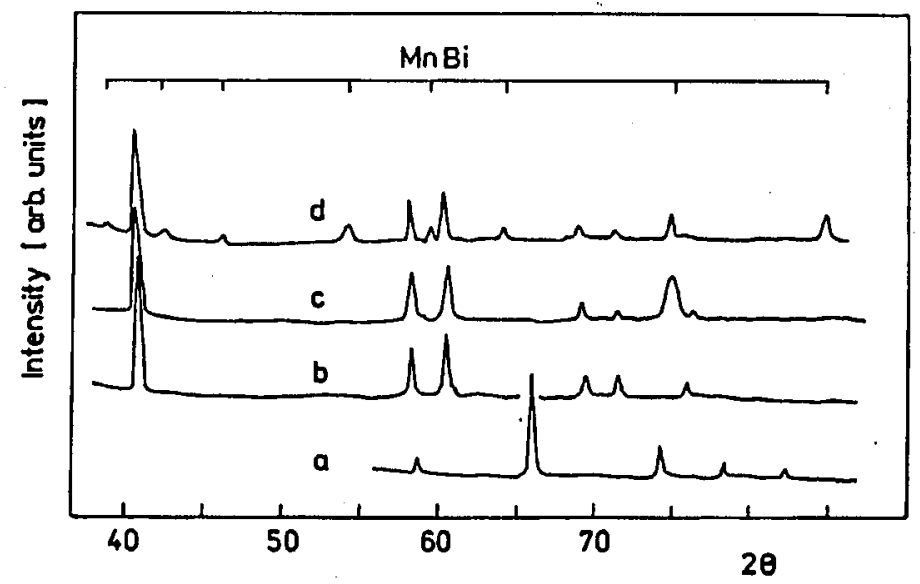

Fig. 1. X-ray diffraction patterns of as-milled, mechanically alloyed $\mathrm{MnBi}$ powders. 


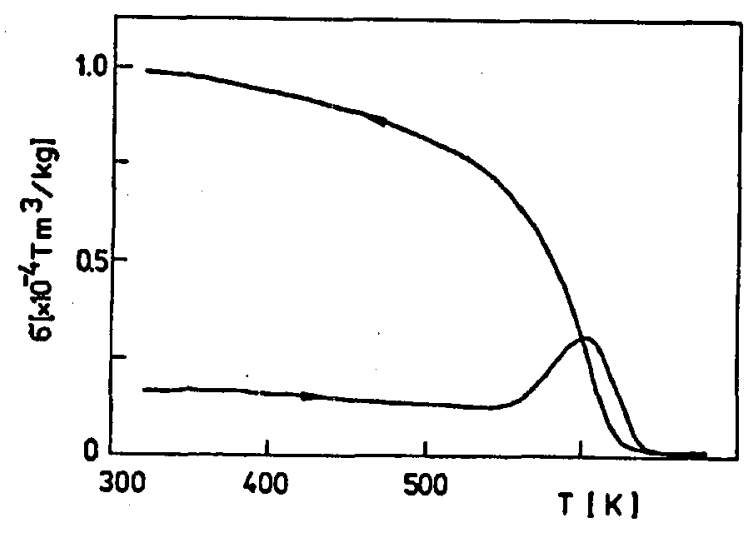

Fig. 2. The thermomagnetic curves for MnBi mechanically alloyed after $120 \mathrm{~h}$ milling.

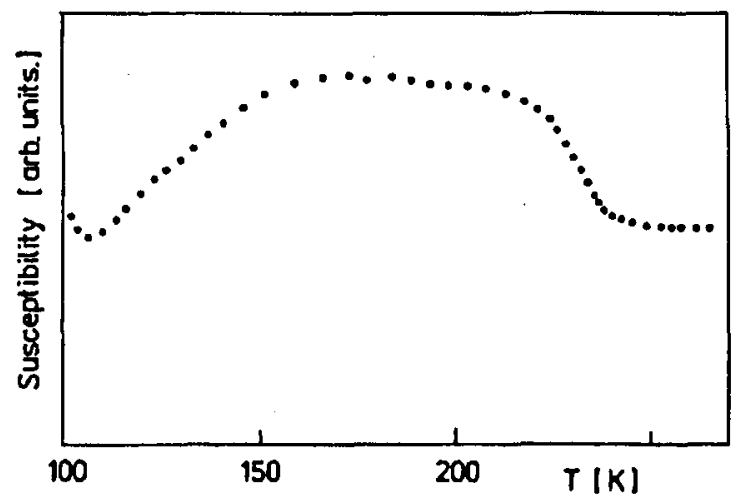

Fig. 3. Temperature dependence of the measured initial susceptibility of MnBi powders after mechanical alloying.

$a$ and $b$ ) are shown for comparison. It can be seen from the curve $c$ that the line $(2 \Theta=75$ degs $)$ due to a fine microstructure of $\mathrm{MnBi}$ has decreased and has been converted (curve $d$ ) into a hexagonal phase. Shultz et al. [4] observed a similar change in the X-ray diffraction (XRD) patterns from $\mathrm{NdFeB}$ samples. Another line in the curve $c$ represents a diamagnetic Bi matrix.

Figure 2 shows the magnetization vs. temperature curves for a $\mathrm{MnBi}$ powder sample. The magnetic phase $\mathrm{MnBi}$ is formed by a heat treatment. Because of the extremely fine microstructure of the milled powders the reaction can take place at relatively low temperature and with short reaction times. The maximum magnetization is obtained after annealing at $573 \mathrm{~K}$ for 20 minutes.

A temperature hysteresis occurs, so that ferromagnetism disappears at $633 \mathrm{~K}$ on heating and reappears at $613 \mathrm{~K}$ on cooling. In these compounds the structures are all the same, only at the temperature the distance between the Mn atoms varying. The fact that the point for Mn lies on the steep part of Bethe's curve 
agrees nicely with the fact that disappearance of ferromagnetism is associated with a sudden contraction of the lattice. Magnetic measurcments were made in a magnetic field parallel to the alignment axis. The intermetallic compound $\mathrm{MnBi}$ is ferromagnetic with the saturation moment of about $1.0 \times 10^{-4} \mathrm{~T} \mathrm{~m} / \mathrm{kg}$. The equilibrium phase at room temperature is hexagonal and is generally known in the recent literature as the low-temperature phase (LTP) to distinguish it from a high-temperature phase (IITP) stable above $613 \mathrm{~K}$ [5]. The structure and composition of the IITP are slightly different from the LTP. In our samples the third phase exists at low temperature. This phase is referred to as the new phase (NP) or as the high coercive (IIC) phase of the MnBi. NP phase has the Curie temperature of $513 \mathrm{~K}$. Our initial susceptibility data in Fig. 3 indicate that both the phases coexist in at least some of the particles. In the samples frozen at moderate rates, only a part of the $\mathrm{Mn}$ is presented as LTP $\mathrm{MnBi}$. At least a part of the remaining $\mathrm{Mn}$ is present in the NP form, which is ferromagnetic with the saturation moment comparable to LTP [6].

\section{Conclusions}

The present work has demonstrated that mechanical alloying is an effective technique of the synthesis of intermetallic compound $\mathrm{MnBi}$.

\section{References}

[1] T. Chen, I. Appl. Phys. 45, 2358 (1974).

[2] M.R. Notis, D.M. Shah, S.P. Young, C.D. Grahtam Jr, IEEE Trans. Magn. MAG-15, 957 (1979).

[3] J.S. Benjamin, Sci. Amer. 234, 40 (1976).

[4] L. Schultz, J. Wecker, Sci. Eng. 89, 127 (1988).

[5] T. Chen, W. Stitius, IEEE Trans. Magn. 10, 581 (1977).

[6] C. Winter, A.S. Arrot, J. Appl. Phys. 53, 8142 (1982). 